\title{
ST Segment Deviation Single Beat
}

National Cancer Institute

\section{Source}

National Cancer Institute. ST Segment Deviation Single Beat. NCI Thesaurus. Code

C117800.

An electrocardiographic measurement of the mean amplitude (usually measured in $\mathrm{mV}$ )

of the ST segment deviation above or below the isoelectric baseline measured from the baseline to the ST segment of a single beat utilizing one or more leads. Based on the recording gain, this measurement may also be reported in $\mathrm{mm}$. (CDISC) 